Sônia Maria da Rocha

\title{
Continente - Saúde: As Tramas do Cotidiano. Um estudo sobre a Saúde do Idoso da Ilha da Conceição.
}

\section{Dissertação de Mestrado}

Dissertação apresentada como requisito parcial para obtenção do grau de Mestre pelo Programa de Pósgraduação em Serviço Social do Departamento de Serviço Social do Departamento de Serviço Social da PUC-Rio.

Orientadora: Profa. Zélia Milanez de Lossio Seiblitz 


\section{Pontificia Universidade Católica $_{\text {mato }}$ DO RIO DE JANEIRO}

\section{Sonia Maria da Rocha}

\section{Continente - Saúde: as tramas do cotidiano. Um estudo sobre a saúde do idoso na Ilha da Conceição.}

Dissertação apresentada como requisito parcial para obtenção do grau de Mestre pelo Programa de Pósgraduação em Serviço Social do Departamento de Serviço Social do Centro de Ciências Sociais da PUCRio. Aprovada pela Comissão Examinadora abaixo assinada.

Profa. Zélia Milanez de Lossio e Seiblitz

Orientador

Departamento de Serviço Social - PUC-Rio

Prof ${ }^{a}$. Myrtes de Aguiar Macêdo

Departamento de Serviço Social - PUC-Rio

Profa. Myriam Lins Barros

UFRJ

Profa. Zélia Milanez de Lossio e Seiblitz Vice-Decana de Pós-Graduação e Pesquisa

Do Centro de Ciências Sociais - PUC-Rio 
Todos os direitos reservados. É proibida a reprodução total ou parcial do trabalho sem autorização da universidade, da autora e do orientador.

\section{Sônia Maria da Rocha}

Graduou-se em Serviço Social pela UFF (Universidade Federal Fluminense), em 1974. Dirigiu o Departamento de Gerontologia da SBGG (Sociedade Brasileira de Geriatria e Gerontologia) - Seção Rio de Janeiro - biênio 1999-2000, e Departamento de Gerontologia da SBGG - Nacional - biênio 2000-2002. Assistente Social da Fundação Municipal de Saúde de Niterói. Participou de diversos Congressos na área da Gerontologia.

Ficha Catalográfica

Rocha, Sônia Maria da

Continente - saúde: as tramas do cotidiano: um estudo sobre a saúde do idoso da Ilha da Conceição / Sônia Maria da Rocha ; orientadora: Zélia Milanez de Lossio Seiblitz. - Rio de Janeiro : PUC, Departamento de Serviço Social, 2004.

73 f. ; $30 \mathrm{~cm}$

Dissertação (mestrado) - Pontifícia Universidade Católica do Rio de Janeiro, Departamento de Serviço Social.

Inclui referências bibliográficas.

1. Serviço Social - Teses. 2. Velhice - Construção social. 3. Saúde. 4. Pertencimento. 5. Territorialidade. I. Seiblitz, Zélia Milanez de Lossio. II. Pontifícia Universidade Católica do Rio de Janeiro. Departamento de Serviço Social. III. Título. 


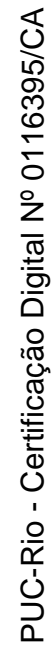

Para Dona Irene, minha mãe, maior inspiração e maior incentivo. 


\section{Agradecimentos}

Aos amigos, com quem trilhei esta caminhada:

Á Professora Zélia Seiblitz, que generosamente orientou-me no sentido de, tal qual José Saramago, a olhar, ver e reparar nas tramas do cotidiano, no tributo à vida.

Ao amigo, José Antônio Fortuna Nogueira (Barroco) pela belíssima parceria e cumplicidade. Sua sensibilidade e respeito com a velhice e com a amizade, tornou esta delicada tarefa de revelar o cotidiano dos idosos, num exercício de valorização da vida.

Willian e Ana, Francisco Werneck e Helena, Satiê, Nelma, Irma, Edite, e tantos outros amigos que se envolveram nas minhas tramas e meus dramas.

Maria Célia Vasconcellos, sempre incentivadora nessa caminhada e compreensiva com minhas faltas.

Aos meus irmãos pelo estímulo e compreensão pela pouca disponibilidade, nos últimos tempos, para os cuidados com a mãe.

Aos amigos da Vice-Presidência de Atenção Coletiva, Ambulatorial e da Família, pelo apoio.

A Zunga e Carlinha pela preciosa ajuda e com quem compartilhei a maior parte desse trabalho.

Aos Professores do Departamento de Serviço Social da PUC-RJ.

À todos os professores e funcionários do Departamento pelos ensinamentos e ajuda.

Às professoras Myrtes de Aguiar Macedo e Myriam Moraes Lins de Barros por aceitarem compor minha banca examinadora.

Aos colegas do curso de Mestrado pelos momentos de afeto, especialmente a Ana Cláudia, Lorena e Nádia com quem tive mais contato.

Aos idosos e profissionais de saúde da Unidade Básica e Médico de Família da Ilha da Conceição, cujos entrevistas permitiram fundamentar esse estudo. 


\section{Resumo}

Rocha, Sônia Maria da; Seiblitz, Zélia Milanez de Lossio. Continente Saúde: As Tramas do Cotidiano. Um estudo sobre a Saúde do Idoso da Ilha da Conceição. Rio de Janeiro, 2003. 96 p. Dissertação de Mestrado Departamento de Serviço Social, Pontifícia Universidade Católica do Rio de Janeiro.

Este trabalho investiga a saúde dos idosos da Ilha da Conceição no município de Niterói, através das representações de saúde e doença dos idosos e dos profissionais de saúde do Programa do Médico de Família e da Unidade Básica de Saúde, serviços oferecidos pela municipalidade. Tem o propósito de subsidiar a avaliação das políticas de saúde e estimular novos estudos capazes de aperfeiçoar instrumentos de intervenção nesta realidade. $O$ estudo situa-se no campo da pesquisa social, utilizando-se o instrumental teórico-metodológico da pesquisa qualitativa. A palavra dos entrevistados é fundamental para a compreensão do processo de construção de saúde no envelhecimento, assim a descrição e a análise das entrevistas valeram-se da mediação de categorias trazidas a partir da constância dos temas abordados pelos entrevistados: a cidadania, a identidade e o pertencimento, as questões existenciais e as relações entre os entrevistados e os serviços de saúde, conceitos desenvolvidos para melhor compreensão do objeto. $\mathrm{O}$ estudo apontou que a concepção de saúde está fortemente influenciada pela cultura local onde o sentimento de pertencimento e da territorialidade, características singulares da Ilha da Conceição, transcendem a dimensão individual. Nessa configuração, a Ilha revelou-se como um continente-saúde, uma comunidade com expressão própria, que assume suas demandas e a sua cidadania.

\section{Palavras-chave} cidadania.

Velhice; construção social da saúde; pertencimento; territorialidade; 


\section{Abstract}

Rocha, Sônia Maria da; Seiblitz, Zélia Milanez de Lossio (Advisor): Continente - Saúde: As Tramas do Cotidiano. Um estudo sobre a Saúde do Idoso da Ilha da Conceição. Rio de Janeiro, 2003. 96p. MSc. Dissertation - Departamento de Serviço Social, Pontifícia Universidade Católica do Rio de Janeiro.

This work studies the health of the elderly of Ilha da Conceição, a neiborghood in Niterói, through the elderly health and disease representatives and also by the health professionals of the program called Programa do Médico de Família e da Unidade Básica de Saúde, services offered by the municipality. The aim of this program is to support the assessment of health policies and encourage new studies to improve devices for intervening in this scenario. This study lies in the field of social research using theoretical and methodological devices of qualitative research. The word of the interviewees is basic to comprehension of the elderly health building process, therefore, the description and analysis of the interviews have taken advantage of the mediation of some categories which have been brought into focus by the interviewees who frequenty mentioned subjects such as citizenship, identity and inclusion, existential issues and the relationship between the interviewees and health services-concepts developed for a better understanding of the objective. This study pointed out to a health concept which is strongly influenced by the local culture where the feeling of belonging to those people and to that place are unique features of Ilha da Conceição and transcends the individual dimension. Taking all those characteristics, Ilha-island- has revealed itself as a health-continent, a community with a particular expression, once it commits itself to accomplishing the community demands and its citizenship.

\section{Keywords}

Elderly; social building of health; inclusion; territory feeling; citizenship. 


\section{Sumário}

$\begin{array}{ll}\text { 1. Introdução } & 10\end{array}$

2. A Velhice como Categoria Sociológica: Uma Trajetória 17

2.1 Panorama da temática da velhice no Brasil 17

2.2 Os movimentos sociais e a politização da velhice 23

3. Reformulações no Setor de Saúde 30

3.1 Movimentos precursores da consolidação do SUS 30

3.2 Políticas de Saúde e assistência à população idosa: 33

3.3 Indicadores sociais como base para a formulação das políticas de saúde

4. A Construção da velhice na Ilha da Conceição 42

4.1 A llha e suas particularidades 42

4.2 O que dizem as falas dos idosos da Ilha da Conceição 46

a) - Cidadania

b) - Questões existenciais e o momento da velhice

c) - Identidade e pertencimento

d) - Relações dos idosos com o sistema de saúde

4.3 A fala dos profissionais de Saúde: outros interlocutores nesse estudo

5. Considerações Finais 64

6. Referências Bibliográficas 70

$\begin{array}{ll}\text { 7. Anexos } & 73\end{array}$ 
".... a palavra é o modo mais puro e sensivel de relação social."

(Bakhtin, apud Minayo,2000) 